\title{
LIQUID VIBRATIONS IN CYLINDRICAL TANKS WITH AND WITHOUT BAFFLES UNDER LATERAL AND LONGITUDINAL EXCITATIONS
}

\author{
E. STRELNIKOVA* \\ A. Podgorny Institute of Mechanical Engineering Problems \\ of the Ukrainian Academy of Sciences \\ UKRAINE \\ V.N. Karazin Kharkiv National University \\ UKRAINE \\ E-mails: elena15@gmx.com; basil@ipmach.kharkov.ua; estrel@ipmach.kharkov.ua \\ D. KRIUTCHENKO and V. GNITKO \\ A. Podgorny Institute of Mechanical Engineering Problems \\ of the Ukrainian Academy of Sciences \\ UKRAINE \\ A. TONKONOZHENKO \\ M.K. Yangel Yuzhnoye State Design Office \\ UKRAINE

\begin{abstract}
The paper is devoted to issues of estimating free surface elevations in rigid cylindrical fluid-filled tanks under external loadings. The possibility of baffles installation is provided. The liquid vibrations caused by lateral and longitudinal harmonic loadings are under consideration. Free, forced and parametrical vibrations are examined. Modes of the free liquid vibrations are considered as basic functions for the analysis of forced and parametric vibrations. The modes of the free liquid vibrations in baffled and un-baffled cylindrical tanks are received by using single-domain and multi-domain boundary element methods. Effects of baffle installation are studied. The problems of forced vibrations are reduced to solving the systems of second order ordinary differential equations. For parametric vibrations the system of Mathieu equations is obtained. The numerical simulation of free surface elevations at different loadings and baffle configurations is accomplished. Beat phenomena effects are considered under lateral harmonic excitations. The phenomenon of parametric resonance is examined under longitudinal harmonic excitations.
\end{abstract}

Key words: sloshing, cylindrical tank, baffle, free and forced vibrations, parametric resonance, boundary element method, Ince-Strutt diagram.

\section{Introduction}

Thin-walled structures are widely used in different engineering areas: chemical and aerospace industries, transportation, oil and gas producing. Usually these structures operate at intensive thermal and stress loadings, in interaction with fluids located in their containers. Many issues and challenges arise in design, and impact simulation of liquid storage tanks. Failures of these tanks, following destructive earthquakes or explosives, may lead to environmental catastrophes, loss of valuable contents, and disruption of fire-fighting efforts. Liquid sloshing often occurs when extreme loads are applied to the structures with

\footnotetext{
* To whom correspondence should be addressed
} 
compartments partially filled with different liquids. So the topical issue here is a problem of estimating frequencies and modes of vibrations of such facilities. The first research devoted to the fluid-structure interaction appeared in the 1960ies when the first missions to Jupiter failed. Among other studies the paper of Kumar [1] should be mentioned. Further research works were devoted to free vibrations of prismatic and cylindrical tanks in 2D formulations (Biswal et al. [2]). Liquid sloshing also a causes damage of roofs and upper walls of storage tanks. Many inadequately designed tanks and liquid storages have been damaged in extensive earthquakes.

Sloshing frequencies and mode shapes for two- and three-dimensional rectangular tanks were obtained by Abramson [3] by the method of variables separation. A comprehensive review of sloshing phenomenon was made by Ibrahim [4]. It should be noted that it is difficult or impossible to obtain analytic solutions for tanks and reservoirs of complicated geometrical shapes. So, a lot of numerical methods have been employed for solving linear boundary value problems of liquid sloshing. Ru-De [5] presented finite element methods for analysis of linear liquid sloshing in the upright cylindrical tank under lateral excitations. Note that such 3-D finite element analysis of fluid-structure-soil interaction in nonlinear statement is complex and extremely time consuming. Several simplified theoretical investigations were conducted, eg. By Cho et al. [6], and other numerical methods were elaborated. Faltinsen and Timokha [7] developed analytical linear multimodal methods to analyze liquid slosh in spherical tanks. The volume-of-fluid method was developed by Kim and Lee [8] and Kim et al. [9]. The dynamic analysis of fluid-filled shell structures using coupled finite and boundary element methods was discussed in [10-11]. To damp the liquid motion, reduce structural loads and prevent instability a lot of slosh-suppression devices have been proposed (Choudhary and Bora [12]). These devices are rigid or elastic ring baffles of different sizes and orientation, vertical partitions, various plates partly covering the free surface [13-14]. Design of such devices also requires high accuracy in estimating the sloshing characteristics. Usually, the effect of baffles can be seen only after their installation. But such an experimental work is too expensive. So the development of computational technologies for qualified numerical simulation of baffle influence is a very topical issue. One of the pioneering papers in the area was written by Miles [15]. The effect of baffles on the natural sloshing frequencies was also studied by Gedikli and Erguven [16] with the boundary element method. This method has certain advantages and gives new qualitative possibilities in modeling fluid-structure interaction process (Brebbia et al. [17]). In the basic equations the functions and their derivatives are defined on the domain boundaries only. This allows us to reduce drastically the dimension of the problem. But matrixes of the linear algebraic systems of equations obtained at discretization are dense and non-symmetric, and it leads to increasing the computational costs. It was the reason why multi-domain or macro-element methods based on using boundary elements have been elaborated for solving boundary value problems [17-19]. The main idea of the multi-domain approach consists in dividing an initial domain into smaller ones (sub-domains or macro-elements). Auxiliary interface surfaces are involved to divide the domain, and unknowns here are the velocity potential and flux. Continuity conditions are formulated on fictitious boundaries. Then the systems of linear algebraic equations are established for each macro-element and a global system of equations is formed by assembling equations of all macro-elements using consistency conditions in common interface nodes. The multi-domain BEM is especially effective at numerical simulation of sloshing in tanks with baffles $[13,18,19]$.

In spite of the considerable amount of research in the area there are a lot of problems which need to be scrutinized. This paper is devoted to free and forced liquid vibrations in baffled and un-baffled cylindrical tanks filled with an incompressible ideal liquid. Reservoirs with both horizontal and vertical baffles are considered. The lateral and longitudinal harmonic loadings are supposed to be applied to the structures under consideration. Beating effects and parametric resonances are analyzed.

\section{Problem statement}

In this paper, problems connected with liquid vibrations in rigid cylindrical tanks with and without baffles are considered. The reservoirs under consideration are presented in Fig.1. The horizontal or vertical baffles are installed into cylindrical reservoirs. 
At the first stage we obtain modes and frequencies of liquid sloshing in the reservoirs. These modes are used as basic systems for studying the forced and parametric liquid vibrations in reservoirs under lateral and longitudinal loadings.

Suppose that the liquid in the containers is an ideal and incompressible one, and the fluid motion is irrotational. Then its relative velocity $\mathbf{V}$ has a potential $\Phi=\Phi(t, x, y, z)$, so that $\boldsymbol{V}=\nabla \Phi$.

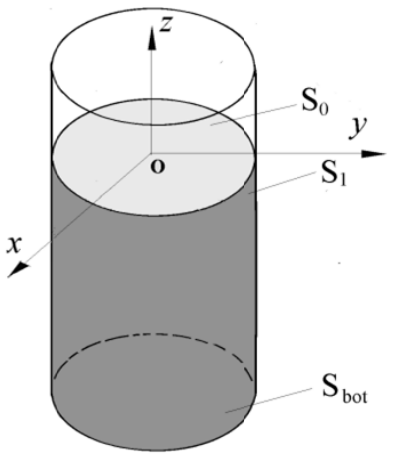

a

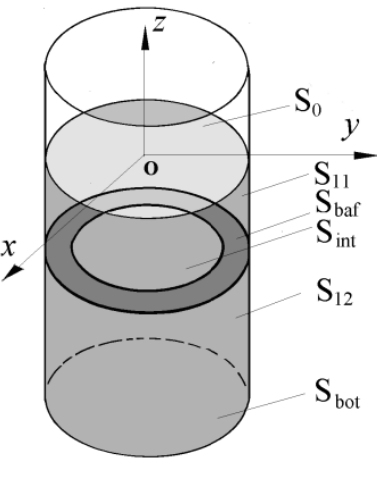

b

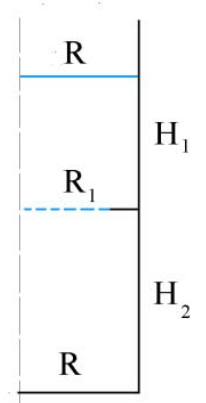

$\mathrm{c}$

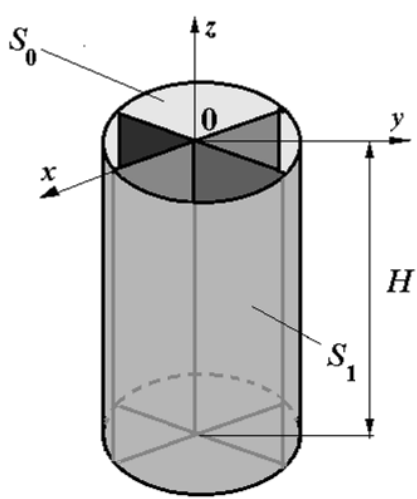

d

Fig.1. Reservoirs partially filled with a liquid.

Let $R$ be the radius, and $H$ be a filling level for all tanks.Designate a moistened shell surface by $S_{l}$, and a free surface by $S_{0}$. Suppose the Cartesian coordinate system $0 x y z$ is connected with containers under consideration, the liquid free surface $S_{0}$ coincides with the plane $z=0$ at the state of rest. Assume that the liquid-filled shell is under vertical and horizontal forces with accelerations $\boldsymbol{a}_{\boldsymbol{x}}$ and $\boldsymbol{a}_{z}$

$$
\boldsymbol{a}_{x}=a_{x}(t) \boldsymbol{i}, \quad \boldsymbol{a}_{\mathrm{z}}=a_{\mathrm{z}}(t) \boldsymbol{k} .
$$

Here the factors $a_{x}(t), a_{z}(t)$ depend on time $t$ only, $\boldsymbol{i}$ and $\boldsymbol{k}$ are the unit vectors along the $O x$ and $O z$ axes.

To begin with, we obtain a relation between a liquid pressure, the velocity potential $\Phi$, and accelerations due to driving forces and gravity. We have

$$
\boldsymbol{a}_{x}=\nabla\left[x \cdot a_{x}(t)\right], \quad \boldsymbol{a}_{z}=\nabla\left[z \cdot a_{z}(t)\right], \quad \boldsymbol{g}=-\nabla(\rho g z)
$$

where $g$ is the gravity acceleration; $x, z$ are coordinates of a point in the liquid.

Equations of motion for the ideal incompressible liquid can be presented in the vector form as follows, Wijngaarden [20]

$$
\boldsymbol{w}+\nabla\left(x a_{x}(t)\right)+\nabla\left(z a_{z}(t)\right)+\nabla(g z)=-\frac{\nabla p}{\rho}
$$

where $\boldsymbol{w}$ is the acceleration of the fluid flow, $\rho$ is the liquid density, and $p$ is the fluid pressure.

So, the acceleration of particles in the liquid under gravitational forces, horizontal and vertical excitations always has a potential (the analog of Prandtl's potential [20]). Using Eq.(2.3) and assuming that the flow is irrotational, the Bernoulli equation is obtained in the following form 


$$
p-p_{0}=-\rho\left[\frac{\partial \Phi}{\partial t}+a_{x}(t) x+\left(g+a_{z}(t)\right) z+\frac{1}{2}|\nabla \Phi|^{2}\right]
$$

where $p_{0}$ is for atmospheric pressure. If small oscillations of the liquid are considered (a linearized formulation is studied), then $|\nabla \Phi|^{2}<<1$, and we have

$$
p-p_{0}=-\rho\left[\frac{\partial \Phi}{\partial t}+a_{x}(t) x+\left(g+a_{z}(t)\right) z\right]
$$

The pressure $p$ on the shell walls in the absence of the horizontal and vertical volume forces is determined from the linearized Bernoulli equation as follows

$$
p-p_{0}=-\rho\left(\frac{\partial \Phi}{\partial t}+g z\right)
$$

From formulas (2.4)-(2.6) one can conclude that evaluating the liquid pressure requires calculating the liquid potential $\Phi$. Assuming the liquid to be inviscid and incompressible, the irrotational fluid motion in the $3 \mathrm{D}$ reservoir is described by the Laplace equation for the velocity potential $\Phi$. This formulation assumes that the fluid remains irrotational if it is irrotational initially.

To determine this potential a mixed boundary value problem for the Laplace equation is formulated. The non-penetration condition on the wetted tank surfaces $S_{l}$ is applied $[10,18]$. On the free surface $(z=0)$ the following dynamic and kinematic boundary conditions must be satisfied

$$
\left.\frac{\partial \Phi}{\partial \boldsymbol{n}}\right|_{S_{0}}=\frac{\partial \zeta}{\partial t}, \quad p-\left.p_{0}\right|_{S_{0}}=0
$$

where an unknown function $\zeta=\zeta(x, y, t)$ describes the shape and position of the free surface. Thus, for the velocity potential we have the following boundary-value problem

$$
\nabla^{2} \Phi=0,\left.\quad \frac{\partial \Phi}{\partial \mathbf{n}}\right|_{S_{I}}=0,\left.\quad \frac{\partial \Phi}{\partial \boldsymbol{n}}\right|_{S_{0}}=\frac{\partial \zeta}{\partial t}, \quad p-\left.p_{0}\right|_{S_{0}}=0
$$

where $p-p_{0}$ is received from Eq.(2.5) at $z=\zeta(x, y, t)$.

The solvability condition for the Neumann problem Eq.(2.8) is given as follows [7]

$$
\iint_{S_{0}} \frac{\partial \Phi}{\partial \mathbf{n}} d S_{0}=0
$$

So, for estimating liquid vibrations in the cylindrical tank depicted in Fig.1a we must solve the boundary value problem (2.8), (2.9).

To estimate the liquid vibrations in the presence of horizontal baffles (Figs 1b, 1c) the multi-domain boundary element method is used [17]. In doing so, we introduce an "artificial" interface surface $S_{\text {int }}$ [13], and divide the region filled with the liquid into two parts, namely: $\Sigma_{1}, \Sigma_{2}$ bounded by surfaces $S_{11}, S_{\text {baf }}, S_{\text {int, }}, S_{\text {bot }}$ and $S_{12}, S_{\text {baf }}, S_{\text {int }}, S_{0}$, respectively. On the interface surface $S_{\text {int }}$ the following boundary conditions are 
specified [17]

$$
\left.\Phi\right|_{S_{\mathrm{int}} \cap \partial \Sigma_{1}}=\left.\Phi\right|_{S_{\mathrm{int}} \cap \partial \Sigma_{2}},\left.\quad \frac{\partial \Phi}{\partial \boldsymbol{n}}\right|_{S_{\mathrm{int}} \cap \partial \Sigma_{1}}=-\left.\frac{\partial \Phi}{\partial \boldsymbol{n}}\right|_{S_{\mathrm{int}} \cap \partial \Sigma_{2}}
$$

So, considering the reservoirs with horizontal baffles we obtain Eqs (2.8)-(2.10) to determine the unknown functions $\zeta=\zeta(x, y, t)$ and $\Phi=\Phi(x, y, z, t)$.

Consider the cylindrical quarter tank (Fig.1d). Using the cylindrical coordinate system $(r, \theta, z)$ we have the following boundary value problem

$$
\nabla^{2} \Phi=0,\left.\frac{\partial \Phi}{\partial r}\right|_{r=R}=0,\left.\frac{\partial \Phi}{\partial z}\right|_{z=-H}=0,\left.\frac{1}{r} \frac{\partial \Phi}{\partial \theta}\right|_{\theta=0, \theta=\frac{\pi}{2}}=0,\left.\frac{\partial \Phi}{\partial \mathbf{n}}\right|_{S_{0}}=\frac{\partial \zeta}{\partial t}, \quad p-\left.p_{0}\right|_{S_{0}}=0
$$

with orthogonality condition (2.9), and $p-p_{0}$ obtained from Eq.(2.5) at $z=\zeta(t, x, y)$.

Consider at first the following auxiliary boundary value problem

$$
\nabla^{2} \Psi=0,\left.\quad \frac{\partial \Psi}{\partial \mathbf{n}}\right|_{S_{I}}=0,\left.\quad \frac{\partial \Psi}{\partial \mathbf{n}}\right|_{S_{0}}=\frac{\partial \zeta}{\partial t}, \quad \frac{\partial \Psi}{\partial t}+\left.g \zeta\right|_{S_{0}}=0, \quad \iint_{S_{0}} \frac{\partial \Psi}{\partial \mathbf{n}} d S_{0}=0 .
$$

Differentiate the forth relation in Eq.(2.12) respect to $t$ and substitute the obtained equality for $\frac{\partial \zeta}{\partial t}$ into the third relation. Further we represent this auxiliary function $\Psi$ as $\Psi(t, x, y, z)=e^{i \chi t} \psi(x, y, z)$, and come to the following eigenvalue problem

$$
\nabla^{2} \psi=0,\left.\quad \frac{\partial \psi}{\partial \mathbf{n}}\right|_{S_{1}}=0, \quad \frac{\partial \psi}{\partial \mathbf{n}}=\left.\frac{\chi^{2}}{g} \psi\right|_{S_{0}}, \quad \iint_{S_{0}} \frac{\partial \psi}{\partial \mathbf{n}} d S_{0}=0
$$

Note that for the cylindrical quarter tank we have $S_{I}=\left\{z=0 \cup r=R \cup \theta=0 \cup \theta=\frac{\pi}{2}\right\}$.

Solving Eqs (2.13) we can obtain the eigenfrequencies and eigenvectors for both un-baffled and baffled tanks with different baffle configurations.

\section{Reducing to systems of boundary integral equations}

\subsection{Shells of revolution without baffles}

The main relation for determining the function $\psi$ from Eq.(2.13) can be written for an arbitrary fluid domain with boundaries $S_{0}, S_{1}$ in the form [17]

$$
2 \pi \psi\left(P_{0}\right)=\iint_{S} \frac{\partial \psi}{\partial \boldsymbol{n}} \frac{1}{\left|P-P_{0}\right|} d S-\iint_{S} \psi \frac{\partial}{\partial \boldsymbol{n}} \frac{1}{\left|P-P_{0}\right|} d S, \quad S=S_{0} \cup S_{1}
$$


Then Eqs (2.13) are reduced to the system of integral equations as in $[10,18]$

$$
\left\{\begin{array}{c}
2 \pi \psi_{1}+\iint_{S_{1}} \psi_{1} \frac{\partial}{\partial \boldsymbol{n}}\left(\frac{1}{\left|P-P_{0}\right|}\right) d S_{1}-\frac{\chi^{2}}{g} \iint_{S_{0}} \psi_{0} \frac{1}{\left|P-P_{0}\right|} d S_{0}+\iint_{S_{0}} \psi_{0} \frac{\partial}{\partial z}\left(\frac{1}{\left|P-P_{0}\right|}\right) d S_{0}=0 \\
-\iint_{S_{1}} \psi_{1} \frac{\partial}{\partial n}\left(\frac{1}{\left|P-P_{0}\right|}\right) d S_{1}-2 \pi \psi_{0}+\frac{\chi^{2}}{g} \iint_{S_{0}} \psi_{0} \frac{1}{\left|P-P_{0}\right|} d S_{0}=0,
\end{array}\right.
$$

where $\left|P-P_{0}\right|$ is the Cartesian distance between points $P$ and $P_{0}$. In Eqs (3.2) for convenience we denote by $\psi_{0}$ the values of potential $\psi$ in the nodes of the free surface $S_{0}$, and by $\psi_{1}$ its values in the nodes of the wetted surfaces $S_{l}$ of the shell.

The following integral operators are introduced

$$
\begin{aligned}
& \boldsymbol{A} \psi_{1}=2 \pi \boldsymbol{I} \psi_{1}+\iint_{S_{1}} \psi_{1} \frac{\partial}{\partial \boldsymbol{n}} \frac{1}{\left|P-P_{0}\right|} d S_{1}, \quad \boldsymbol{B} \psi_{0}=\iint_{S_{0}} \psi_{0} \frac{1}{\left|P-P_{0}\right|} d S_{0}, \\
& \boldsymbol{C} \psi_{0}=\iint_{S_{0}} \psi_{0} \frac{\partial}{\partial z}\left(\frac{1}{\left|P-P_{0}\right|}\right) d S_{0}, \\
& \boldsymbol{D} \psi_{1}=-\iint_{S_{1}} \psi_{1} \frac{\partial}{\partial \boldsymbol{n}} \frac{1}{\left|P-P_{0}\right|} d S_{1}, \quad \boldsymbol{F} \psi_{0}=\iint_{S_{0}} \psi_{0} \frac{1}{\left|P-P_{0}\right|} d S_{0} .
\end{aligned}
$$

With Eqs (3.3) the boundary value problem (2.13) takes the form

$$
\boldsymbol{A} \psi_{1}=\frac{\chi^{2}}{g} \boldsymbol{B} \psi_{0}-\boldsymbol{C} \psi_{0}, \quad P_{0} \in S_{1}, \quad \boldsymbol{D} \psi_{1}=2 \pi \boldsymbol{I} \psi_{0}-\frac{\chi^{2}}{g} \boldsymbol{F} \psi_{0}, \quad P_{0} \in S_{0}
$$

After excluding the function $\psi_{1}$ from Eqs (3.4) we obtain the following eigenvalue problem relative to the potential values $\psi_{0}$ on the free surface only

$$
\left(\boldsymbol{D} \boldsymbol{A}^{-1} \boldsymbol{C}+2 \pi \boldsymbol{I}\right) \psi_{0}-\lambda\left(\boldsymbol{D} \boldsymbol{A}^{-1} \boldsymbol{B}+\boldsymbol{F}\right) \psi_{0}=0, \quad \lambda=\chi^{2} / g
$$

The solution of Eq.(3.5) gives natural modes and frequencies of liquid sloshing in the rigid shell. Consider the boundary value problem (2.8), (2.9) for an arbitrary shell of revolution. In the cylindrical coordinates system $(r, z, \theta)$ we have

$$
\Phi=\sum_{\alpha=0}^{N} \cos \alpha \theta \sum_{k=1}^{M} \dot{d}_{\alpha k}(t) \phi_{\alpha k}(r, z) .
$$

Here $\alpha$ is harmonica number, indexes $\alpha k$ are mode numbers corresponding to $\alpha$. Thus, frequencies and modes of free vibrations are considered separately for different values of $\alpha$. From Eqs (2.7) and (3.6) the 
following expression is received for the free surface elevation

$$
\zeta=\frac{1}{g} \sum_{\alpha=0}^{N} \cos \alpha \theta \sum_{k=1}^{M} \chi_{\alpha k}^{2} d_{\alpha k}(t) \phi_{\alpha k}(r, 0) .
$$

Let $\Gamma$ be a generator of the surface $S_{l}$. Reducing integrals in Eqs (3.3) to one-dimensional ones we obtain the system of one-dimensional integral equations as in Gnitko et al. [18]. For integrals in Eqs (3.3) we have for each harmonic $\alpha$

$$
\begin{aligned}
& \iint_{S_{1}} \psi_{1} \frac{\partial}{\partial \boldsymbol{n}}\left(\frac{1}{\left|P-P_{0}\right|}\right) d S_{1}=\int_{r} \psi_{l}(z) \Theta\left(z, z_{0}\right) r(z) d \Gamma, \quad \iint_{S_{0}} \psi_{0} \frac{1}{\left|P-P_{0}\right|} d S_{0}=\int_{0}^{R} \psi_{0}(r) \Phi\left(P, P_{0}\right) r d r \\
& \Theta\left(z, z_{0}\right)=\frac{4}{\sqrt{a+b}}\left\{\frac{1}{2 r}\left[\frac{r^{2}-r_{0}^{2}+\left(z_{0}-z\right)^{2}}{a-b} \mathrm{E}_{\alpha}(k)-\mathrm{F}_{\alpha}(k)\right] n_{r}+\frac{z_{0}-z}{a-b} \mathrm{E}_{\alpha}(k) n_{z}\right\}, \\
& \Phi\left(P, P_{0}\right)=\frac{4}{\sqrt{a+b}} \mathrm{~F}_{\alpha}(k) ; \quad \mathrm{E}_{\alpha}(k)=(-1)^{\alpha}\left(1-16 \alpha^{2}\right) \int_{0}^{\pi / 2} \cos 2 \alpha l \psi \sqrt{1-k^{2} \sin ^{2} \psi} d \psi, \\
& \mathrm{F}_{\alpha}(k)=(-1)^{\alpha} \int_{0}^{\pi / 2} \frac{\cos 2 \alpha l \psi d \psi}{\sqrt{1-k^{2} \sin ^{2} \psi}}, \quad a=r^{2}+r_{0}^{2}+\left(z-z_{0}\right)^{2}, \quad b=2 r r_{0}, \quad k^{2}=\frac{2 b}{a+b}, \quad l=1 .
\end{aligned}
$$

Evaluation of integral operators in Eq.(3.8) is carried out by the method proposed in [18], [19]. From Eqs (3.5), (3.8) the basic functions $\varphi_{\alpha k}$ are received. Substitute them into expressions (3.6) for the velocity potential and Eq.(3.7) for the free surface elevation.

With Eqs (3.6), (3.7) it is established that the functions $\Phi$ and $\zeta$ satisfy the following equations

$$
\nabla^{2} \Phi=0,\left.\quad \frac{\partial \Phi}{\partial \boldsymbol{n}}\right|_{S_{1}}=0,\left.\quad \frac{\partial \Phi}{\partial \boldsymbol{n}}\right|_{S_{0}}=\frac{\partial \zeta}{\partial t}, \quad \iint_{S_{0}} \frac{\partial \Phi}{\partial \boldsymbol{n}} d S_{0}=0
$$

Then insert Eqs (3.6), (3.7) for $\Phi$ and $\zeta$ into the boundary dynamical condition (3.5) for pressure on the free surface at $z=\zeta(t, x, y)$.

As in the cylindrical system of coordinates there is $x=\rho \cos \theta$, we will be interested only in axisymmetric $(\alpha=0)$ and non-axisymmetric $(\alpha=1)$ modes in expressions (3.6), (3.7). Using Eqs (2.5), (3.6), (3.7) we come to the following equation on the surface $\mathrm{S}_{0}$

$$
\sum_{\alpha=0}^{l} \cos \alpha \theta \sum_{k=1}^{M}\left[\ddot{d}_{\alpha k}(t)+\chi_{\alpha k}^{2}\left(1+\frac{a_{z}(t)}{g}\right) d_{\alpha k}(t)\right] \phi_{\alpha k}(r, z)+r \cos \theta a_{x}(t)=0, \quad z=\zeta .
$$

Accomplishing the dot product of Eq.(3.10) and $\varphi_{\alpha l}(\alpha=0,1 ; l=\overline{1, M})$ and having used 
orthogonality of modes, we receive the uncoupled system of ordinary differential equations of the second order

$$
\ddot{d}_{1 k}+\chi_{l k}^{2} d_{1 k}+a_{x}(t) F_{1 k}=0, \quad \ddot{d}_{0 k}+\chi_{0 k}^{2}\left(1+\frac{a_{z}(t)}{g}\right) d_{0 k}=0, \quad F_{1 k}=\frac{\left(r, \phi_{1 k}\right)}{\left(\phi_{1 k}, \phi_{1 k}\right)} ; \quad k=\overline{1, M} .
$$

To solve system Eq.(24) the initial conditions are required.

\subsection{Cylindrical tanks with baffles}

The singular integral equations for the shell of revolution with ring baffles were received and described in detail by the authors in $[11,18]$. It should be noted that the system of second order differential equations in this case has also the form Eqs (3.11), but with other modes and frequencies.

Consider the quarter tank, Fig.1d. According to Ibrahim [4] we assume that for the shell of revolution with two vertical baffles we can seek the potential $\Phi$ as follows

$$
\Phi=\sum_{\alpha=0}^{N} \cos 2 \alpha \theta \sum_{k=1}^{M} \dot{d}_{\alpha k}(t) \phi_{\alpha k}(r, z)
$$

Using this relation we satisfy boundary conditions from Eqs (2.11) on the vertical baffles. The system of singular integral equations for $\varphi_{\alpha k}(r, z)$ acquires the form analogical to system (3.2), (3.8), but in Eqs (3.8) we set $l=2$. Note that the system of second order differential equations has again the form (3.11), but with other expressions for modes and frequencies.

\section{Free vibrations of the liquid in un-baffled and baffled cylindrical tanks}

Vibrations in an oscillatory system are called free or natural if they occur after the system has been taken out of equilibrium by some initial excitations and then left to itself.

Consider circular fluid-filled cylindrical shells of radius $R$ with a flat bottom. The filling level in all shells is denoted by $H$. The installation of horizontal or vertical baffles is supposed. The horizontal baffle is considered as a circle flat plate with a central hole with radius $R_{\text {baf }}$ (the ring baffle). The vertical coordinate of the baffle position is $H_{l}\left(H_{l}<H\right)$. The radius of the interface surface is denoted by $R_{l}$ (see Fig.1c), and for the filling level we have $H=H_{1}+H_{2}$. Considering the vertical baffles, we suppose that the cylindrical tank is divided by two orthogonal walls as shown in Fig.1d. Numerical evaluations of frequencies for the un-baffled cylindrical shell are provided for validation of the proposed boundary element method (BEM). The accuracy $\varepsilon=10^{-3}$ is achieved with 120 boundary elements along the bottom, 120 elements along wetted cylindrical parts, and 100 elements along the radius of the free surface. Table 1 below provides the numerical and analytical values of the frequency parameter $\omega^{2}=\chi^{2} / g$ and frequencies $\chi$ for liquid sloshing at circumference number $\alpha=1$ supposing $R=1.0 \mathrm{~m}$ and $H=1.0 \mathrm{~m}$.

The analytical solution of Ibrahim [4] is used here for comparison and validation

$$
\frac{\chi_{1 k}^{2}}{g}=\frac{\mu_{1 k}}{R} \tanh \left(\mu_{1 k} \frac{H}{R}\right), \quad \phi_{k}=J_{1}\left(\frac{\mu_{k}}{R} r\right) \cosh \left(\frac{\mu_{k}}{R} z\right) \cosh ^{-1}\left(\frac{\mu_{k}}{R} H\right), \quad k=1,2 \ldots
$$

where values $\mu_{k}$ are roots of the equation 


$$
\frac{d J_{1}(x)}{d x}=2\left[J_{0}(x)-J_{2}(x)\right]
$$

and $J_{0}(x), J_{l}(x), J_{2}(x)$ are Bessel functions of the first kind.

Table 1. Frequencies and frequency parameters for an un-baffled cylindrical shell, $\alpha=1$.

\begin{tabular}{|l|c|c|c|c|c|c|}
\hline \multicolumn{2}{|c|}{$k$} & 1 & 2 & 3 & 4 & 5 \\
\hline \multirow{2}{*}{$\begin{array}{l}\text { Frequency } \\
\text { parameter }\end{array}$} & BEM & 1.750 & 5.332 & 8.538 & 11.709 & 14.870 \\
\cline { 2 - 7 } & Formula (4.1) & 1.750 & 5.331 & 8.536 & 11.706 & 14.864 \\
\hline \multicolumn{2}{|c|}{ Frequency, BEM } & 4.141 & 7.227 & 9.1429 & 10.707 & 12.071 \\
\hline
\end{tabular}

Next, the influence of horizontal baffles is discussed. The natural sloshing frequencies are calculated for $\alpha=1$ at $H_{2}=0.5 \mathrm{~m}, H_{2}=0.9 \mathrm{~m}$, and with $R_{1}=0.7 \mathrm{~m}$. A comparison of results obtained with the proposed multi-domain BEM (MBEM) and the analytically oriented approach presented by Gavrilyuk et al. in [21] is shown in Tab.2.

Table 2. Comparison of analytical and numerical results, $\alpha=1$.

\begin{tabular}{|l|c|c|c|c|c|c|}
\hline Position & method & $n=1$ & $n=2$ & $n=3$ & $n=4$ & $n=5$ \\
\hline$H_{2}=0.5$ & MBEM & 1.3663 & 5.2941 & 8.5359 & 11.7097 & 14.870 \\
\cline { 2 - 7 } & {$[21]$} & 1.3662 & 5.2940 & 8.5357 & 11.7092 & 14.864 \\
\hline$H_{2}=0.9$ & MBEM & 0.7079 & 4.5069 & 8.1947 & 11.5556 & 14.850 \\
\cline { 2 - 7 } & {$[21]$} & 0.7079 & 4.5068 & 8.1945 & 11.5550 & 14.832 \\
\hline
\end{tabular}

In Tab.3 the frequencies of liquid vibrations are listed for an un-baffled cylindrical shell (UB), the cylindrical shells with horizontal (HB) and vertical (VB) baffles for $\alpha=0,1$.

Table 3. Frequencies of liquid vibrations in different cylindrical tanks.

\begin{tabular}{|c|c|c|c|c|c|}
\hline$\alpha$ & shell type & $n=1$ & $n=2$ & $n=3$ & $n=4$ \\
\hline \multirow{4}{*}{0} & $\mathrm{UB}$ & 6.1248 & 8.2919 & 9.98475 & 11.4295 \\
\cline { 2 - 6 } & $\mathrm{HB}, H_{2}=0.5$ & 6.0654 & 8.2896 & 9.98475 & 11.4295 \\
\cline { 2 - 6 } & $\mathrm{HB}, H_{2}=0.9$ & 4.7242 & 7.7948 & 9.70352 & 11.2043 \\
\cline { 2 - 6 } & $\mathrm{VB}$ & 6.1248 & 8.2919 & 9.98475 & 11.4295 \\
\hline \multirow{4}{*}{1} & $\mathrm{UB}$ & 4.1424 & 7.2286 & 9.14726 & 10.7120 \\
\cline { 2 - 6 } & $\mathrm{HB}, H_{2}=0.5$ & 3.6520 & 7.2028 & 9.14565 & 10.7120 \\
\cline { 2 - 6 } & $\mathrm{HB}, H_{2}=0.9$ & 2.6340 & 6.6452 & 8.96218 & 10.6436 \\
\cline { 2 - 6 } & $\mathrm{VB}$ & 5.4582 & 8.1067 & 9.87918 & 12.6574 \\
\hline
\end{tabular}

The frequencies of reservoirs with horizontal baffles are smaller compared to un-baffled ones for $\alpha=0,1$. Installation of vertical baffles moves the spectrum of resonant frequencies towards high frequencies oscillations for $\alpha=1$ and does not influence axisymmetric frequencies, $\alpha=0$. Results presented here may be used for resonant frequency turning. 


\section{Forced and parametric liquid vibrations}

\subsection{Cylindrical shells under lateral harmonic excitations}

Cylindrical shells with and without baffles are considered. Hereinafter, for all the shells we suppose that the radius is $R=1 \mathrm{~m}$, and filling level is $H=1 \mathrm{~m}$. The tank is subjected to periodic loading $a_{x}(t)=a_{1} \cos \omega t$ that is applied in the horizontal direction. So, the only non-axisymmetric modes with $\alpha=1$ are under consideration. Suppose that before applying the horizontal loading the reservoir was at the state of rest. So, we need to solve system (3.11) under zero initial conditions. It follows from Eqs (3.11) that under these initial conditions we obtain

$$
d_{1 k}(t)=\frac{a_{1} F_{l k}}{\chi_{l k}^{2}-\omega^{2}}\left(\cos \omega t-\cos \chi_{l k} t\right), \quad F_{l k}=\frac{\left(r, \phi_{1 k}\right)}{\left(\phi_{1 k}, \phi_{l k}\right)}, \quad k=\overline{1, M}
$$

To obtain the free surface elevation we use Eq.(3.7). Figures $2 a$ and $2 b$ show time-histories of the free surface elevation at the points $\rho=0.5 R, z=\zeta$ and $\rho=R, z=\zeta$, respectively, at $\theta=0, \omega=3 \mathrm{~Hz}, a_{1}=1$ during $10 \mathrm{sec}$.

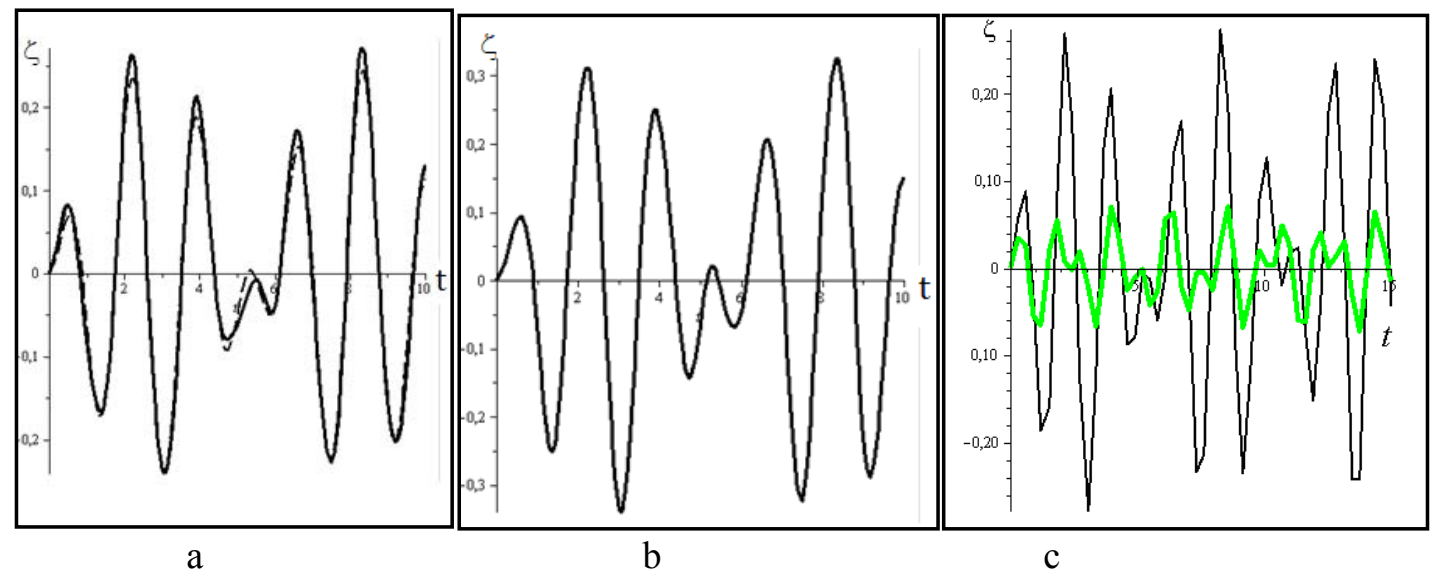

Fig.2. Time-histories of the free surface elevation.

It should be noted that convergence is achieved when $M=4$, Eq.(3.7). Figure 2a demonstrates differences in $\zeta$ at $M=2$ (dashed line) and $M=4$ (solid line). Results for $M=4$ and $M=5$ practically coincided. Consider the excitation $a_{x}(t)=\cos 3 t$. Its frequency is apart from the eigenfrequencies of both the un-baffled shell and the shell with vertical baffles. Figure $2 \mathrm{c}$ shows an essential decrease in the liquid sloshing amplitude in the presence of vertical baffles. The black line here corresponds to the cylindrical shell without baffles and the green line corresponds to the free surface elevation in the presence of vertical baffles at the point $\theta=0, \rho=R, z=\zeta$ during $15 \mathrm{sec}$.

Figures $3 \mathrm{a}$ and $3 \mathrm{~b}$ show time-histories of the free surface elevation at the points $\theta=0$, $\rho=0.5 R, z=\zeta$ and $\theta=0, \rho=R, z=\zeta$, respectively, at $\omega=4 \mathrm{~Hz}$ during $50 \mathrm{sec}$. 


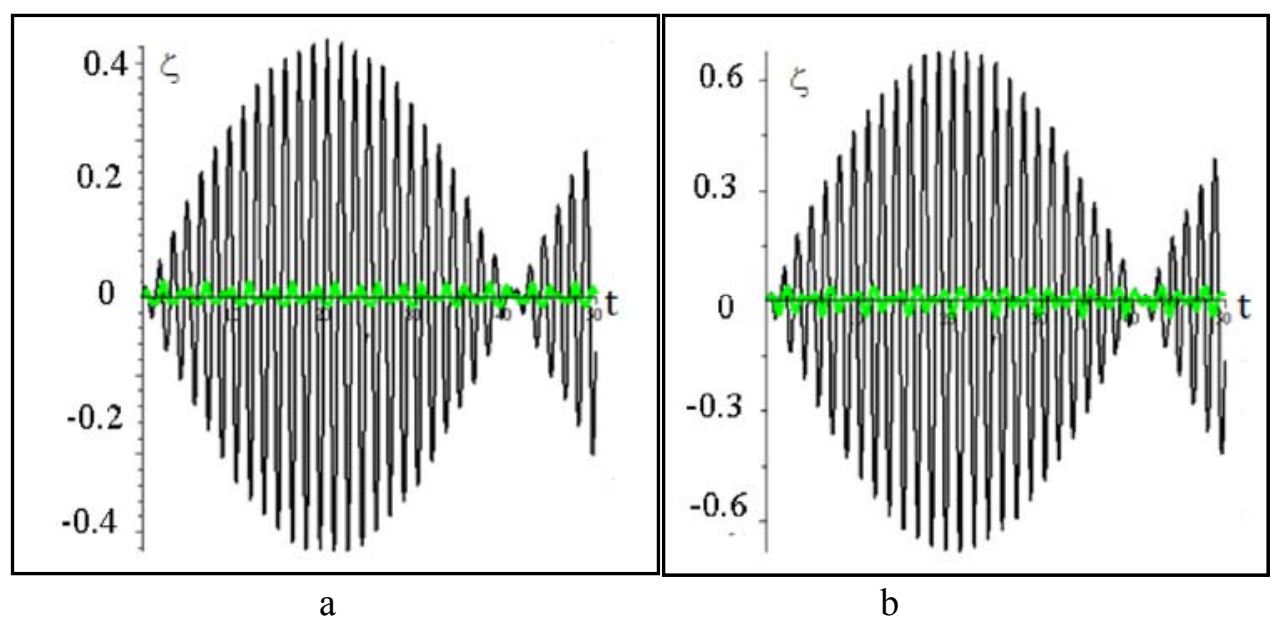

Fig.3. Time-histories of the free surface elevation, at $\omega=4 \mathrm{~Hz}$.

The green lines here correspond to the free surface elevation in the presence of the horizontal baffle $\left(H_{2}=0.9 m, R_{1}=0.7 m\right)$. From the results obtained one can conclude that when excitation frequency $\omega=4 \mathrm{~Hz}$ approaches the first natural sloshing frequency $\omega=4.14 \mathrm{~Hz}$ the beat phenomenaeffect takes place. After the baffle installation the oscillation amplitudes decrease drastically. It should be noted that the amplitudes of the free surface elevation in the un-baffled tank at beating are very large, so the problems in nonlinear formulation need to be considered. Note that for the cylindrical quarter tank, Fig.1d, the beat phenomena with smaller amplitudes occur when the excitation frequency is near $\omega=5.5 \mathrm{~Hz}$.

\subsection{Cylindrical shells under longitudinal harmonic excitations}

Consider shells depicted in Fig.1 under longitudinal harmonic excitations $a_{z}(t)=-a_{2} \cos \omega t$ only. Then system (3.11) is transformed to uncoupled Mathieu equations

$$
\ddot{d}_{0 k}+\chi_{0 k}^{2}\left(1-\frac{a_{2} \cos \omega t}{g}\right) d_{0 k}=0, \quad k=\overline{1, M}
$$

The parametric vibrations of baffled and un-baffled cylindrical shells caused by longitudinal excitations $a_{z}(t)=-a_{2} \cos \omega t$ at different excitation frequencies $\omega$ are examined. Figures $4 \mathrm{a}, 4 \mathrm{c}, 4 \mathrm{e}$ correspond to time histories of the free surface elevation and Figs $4 \mathrm{~b}, 4 \mathrm{~d}, 4 \mathrm{f}$ present phase portraits of unbaffled shells in coordinates $\zeta, \zeta_{t}^{\prime}$ at the point $\rho=0, z=\zeta$ at $\dot{d}_{01}(0)=0.05, \dot{d}_{0 k}(0)=0.0$, $k=\overline{2, M}, d_{0 k}(0)=0, k=\overline{1, M}$. Here and hereinafter green lines are graphs of the vertical excitations, by numbers 1 and 2 the time-dependent graphs of free surface elevations of un-baffled cylindrical tanks (Fig.1a) and tanks with horizontal baffles (Fig. $1 \mathrm{~b}$ ) are marked off. The parameters of baffle are $H_{2}=0.9 \mathrm{~m}, R_{1}=0.7 \mathrm{~m}$. It has been ascertained that the influence of vertical baffles (Fig.1d) is not sufficient in the case of longitudinal excitations.

Equations (5.2) are well-known and their solutions have been the subject of a lot of research. In this paper, the algebraic method for their solution is in use [22].

It should be noted that as against the forced lateral excitations with zero initial conditions for all unknowns, in the case of parametric vibrations it is necessary to set at least one nonzero initial value for receiving nonzero solutions of Eqs (5.2). 


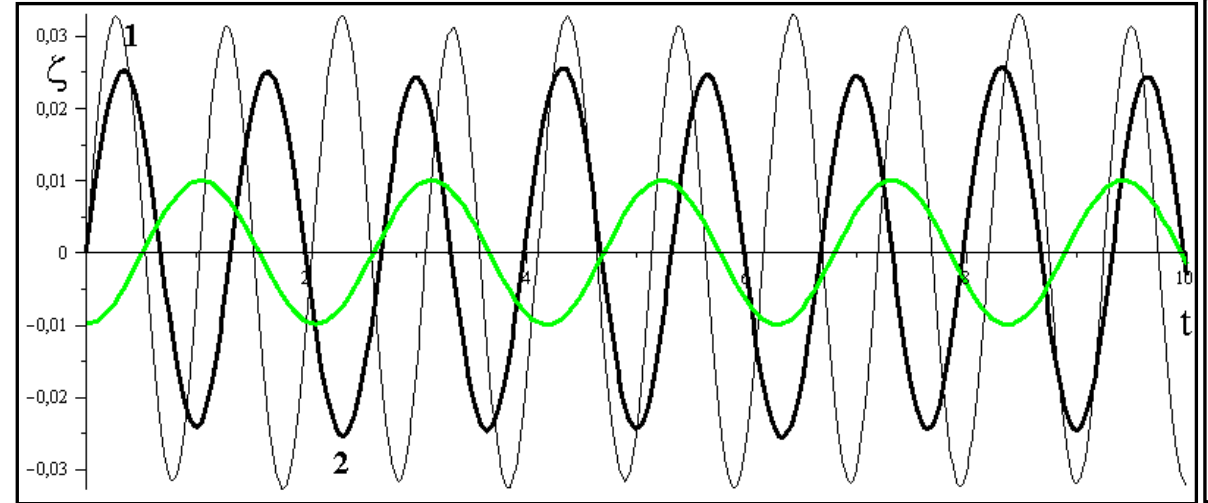

a

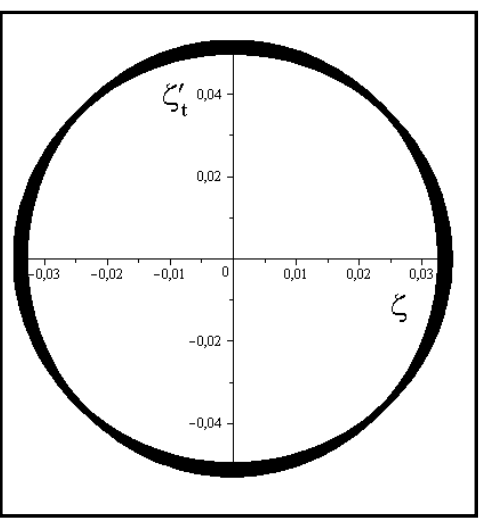

b

$\omega=3 \mathrm{~Hz}, a_{2}=1$

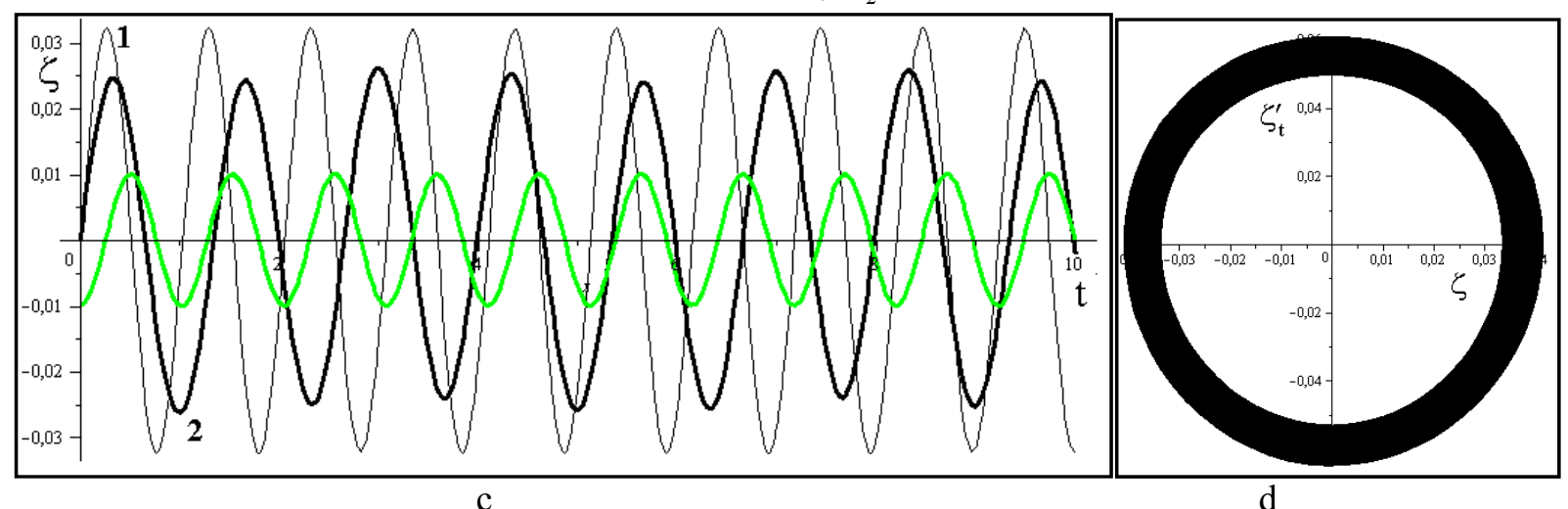

$\omega=6.125 \mathrm{~Hz}, a_{2}=1$

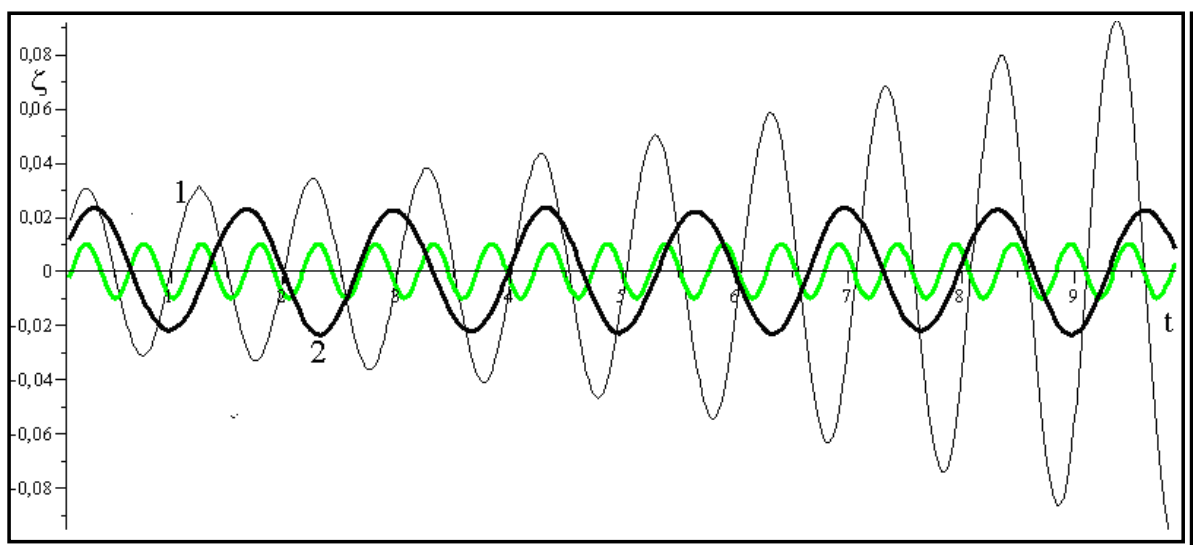

$$
\omega=12.25 \mathrm{~Hz}, a_{2}=1
$$

Fig.4. Time histories and phase portraits of the free surface elevation.

The free surface oscillation under vertical harmonic excitation is the parametric sloshing. Therefore, some unstable vibrations may occur depending on the values of parameters. The sub-harmonic (or principle) parametric resonance occurrs when the liquid container is vertically excited at the frequency close to the doubled natural sloshing frequency. It leads to an infinitel increase in the free surface elevation (Figs 4e, 4f).

It should be noted that $\omega=6.125 \mathrm{~Hz}$ is the first natural frequency of the un-baffled tank. Analyzing the phase portrait corresponding to this frequency one can conclude that the system is near loosing its stability, but the time history shows a periodic behaviour (Fig.4c) For the near twice natural frequency of the 
free surface the oscillations caused by the periodic excitation acquire an increasing character (Figs 4e, 4f).

For a more detailed estimation of stability regions we use the Ince-Strutt diagram in the form presented by Butikov [23]. For this purpose we transform Eqs (29) as follows

$$
\frac{d^{2} u}{d \tau^{2}}+\kappa(1-\mu \cos \tau) u=0, \quad \tau=\omega t, \quad \kappa=\frac{\chi_{k}^{2}}{\omega^{2}}, \quad \mu=\frac{a_{2}}{g}, \quad u=d_{0 k}, \quad k=\overline{1, M}
$$

The Ince-Strutt diagram is shown in Fig.5. Here the following analytical expressions for curves 1-5 are obtained as Butikov [23]

$$
\begin{aligned}
& \mu_{1}(\kappa)=2 \sqrt{\kappa(\kappa-1)(\kappa-4) /(3 \kappa-8)}, \quad \kappa<0, \\
& \mu_{2}(\kappa)=\frac{1}{4} \sqrt{(9-4 \kappa)(13-29 \kappa)}-(9-4 \kappa), \quad 0<\kappa<1 / 4, \\
& \mu_{3}(\kappa)=\frac{1}{4}(9-4 \kappa \mp \sqrt{(9-4 \kappa)(13-29 \kappa)}), \quad 1 / 4<\kappa<13 / 20, \\
& \mu_{4}(\kappa)=\sqrt{2(\kappa-1)(\kappa-4)(\kappa-9) /(\kappa-5)}, \quad 13 / 20<\kappa<1, \\
& \mu_{5}(\kappa)=2 \sqrt{\kappa(\kappa-1)(\kappa-4) /(3 \kappa-8)}, \quad \kappa>1 .
\end{aligned}
$$

These curves divide the plane $(\kappa, \mu)$ into regions corresponding to unstable motions (shaded areas) and stable motions that remain bounded during time (white areas).

Then for results depicted in Fig. 4 we obtain the following coordinates $(\kappa, \mu)$ of points $(A, B, C)$ in the Ince-Strutt diagram for cases under consideration: $\mathrm{A}(2.34 ; 0.102) ; \mathrm{B}(0.25 ; 0.102) ; \mathrm{C}(1.00 ; 0.102)$. Points (A, B, C) correspond to results presented in Figs 4a,4b; Figs 4c,4d; Figs 4e,4f, respectively. Point $C$ is on the border of stability regions, so if we consider, for example, $\mu=0.6$, then point $\mathrm{D}$ with coordinates $\mathrm{D}(1.00$; $0.6)$ is in the area of instability. The graphs in Fig.6 show the free surface elevation at this point in un-baffled (thin line 1) and baffled (thick line 2) reservoirs.

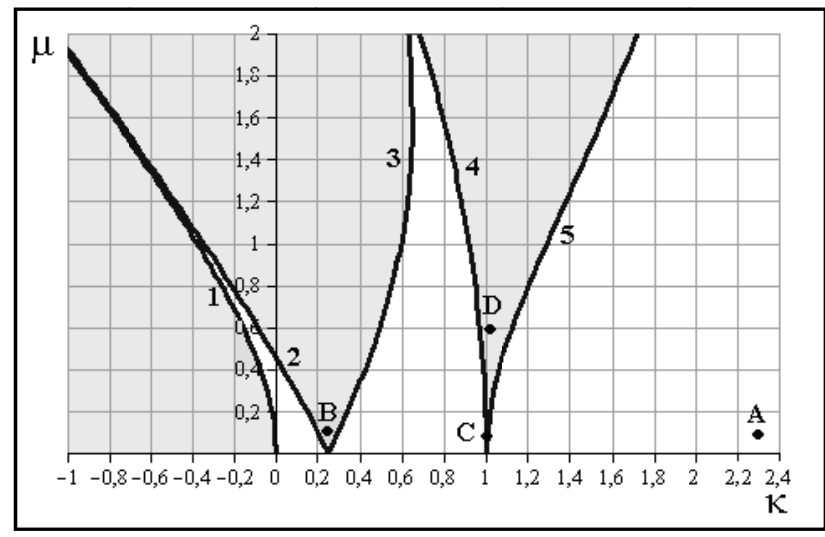

Fig.5. Ince-Strutt diagram.

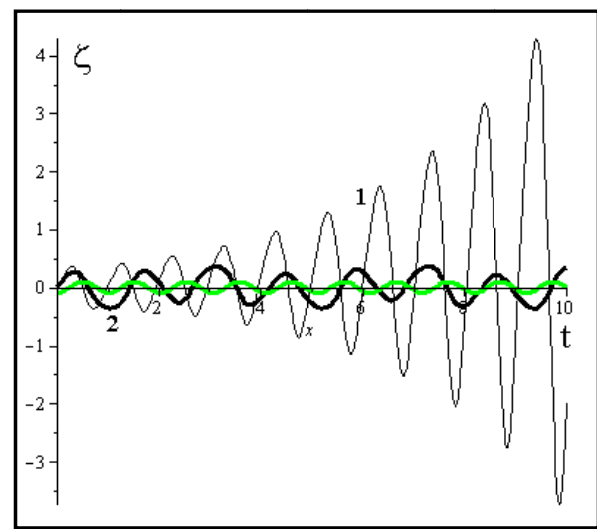

Fig.6. Unstable motion in point D. 
Consider simultaneous actions of lateral and longitudinal excitations. The presence of internal parametric resonance results in a considerable disturbance of non-axisymmetric high-frequency modes in these cases. Let $\omega=12.25 \mathrm{~Hz}$ and $a_{x}(t)=a_{1} \cos \omega t, a_{z}(t)=-a_{2} \cos \omega t, a_{1}=a_{2}=1$. In this case the amplitude of the modes $k=5$ for the un-baffled tank (Tab.1) and $k=4$ for the quarter tank (Tab.2) are excited considerably because of resonance effect. So if the initial data for the un-baffled tank are

$$
d_{0 k}(0)=d_{1 k}(0)=\dot{d}_{1 k}(0)=0, \quad k=\overline{1, M}, \quad \dot{d}_{1 k}(0)=0, \quad k \neq 5, \quad \dot{d}_{15}(0)=0.05
$$

then mode $\alpha=1, k=5$ becomes dominant.

Figure $7 \mathrm{a}$ shows the free surface elevation of the un-baffled tank in the absence of longitudinal excitations at the point $\theta=0, \rho=R, z=\zeta, \omega=12.25 \mathrm{~Hz}$. Here the beating effect appeared. Figure $7 \mathrm{~b}$ shows the free surface behaviour at simultaneous actions of lateral and longitudinal excitations under initial conditions (5.5).

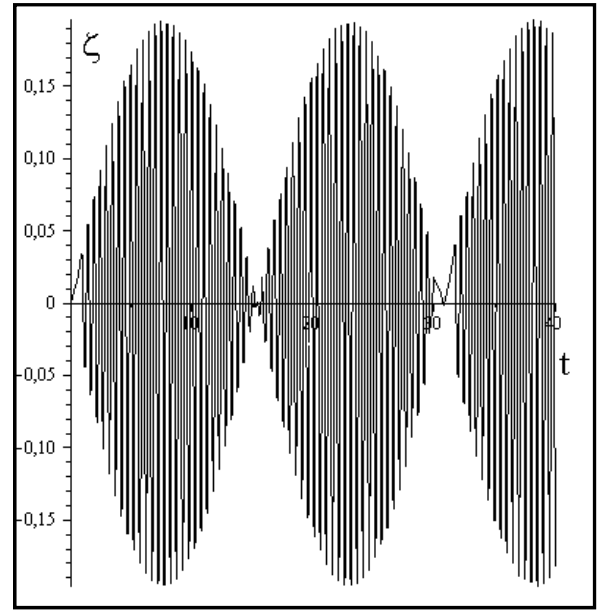

$\mathrm{a}$

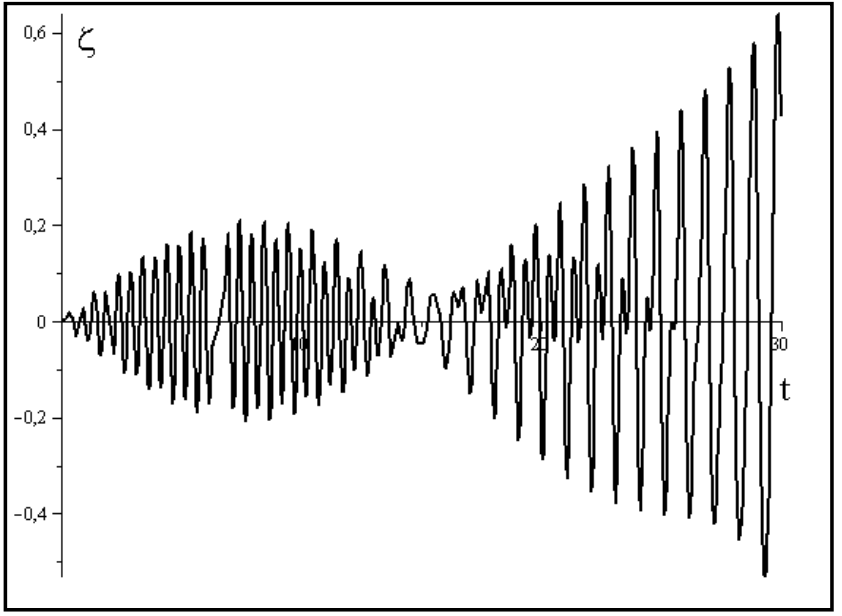

b

Fig.7. Free surface elevation without (a) and with (b) longitudinal excitations.

The motion of the liquid free surface becomes unstable in the presence of longitudinal excitations. This is connected with the essential contribution of the axisymmetric normal mode especially when the excitation frequencies are close to the doubled first natural frequency.

The installation of baffles can be useful for decreasing the free surface elevation. But changing the frequency of forced excitation can produce an undesirable effect consisting in a co-occurence of changed excitation frequency with some natural frequencies of the baffled tank.

\section{Conclusion}

A method is developed that allows estimation of the level of the free surface elevation in cylindrical tanks with and without baffles under longitudinal and lateral loadings. The free, forced and parametric liquid vibrations in cylindrical tanks with and without baffles of equal heights and radii are considered. The benchmark tests are provided for validation of the obtained results. The effects of the baffle installation and their influence on changing the elevation of the free surface are taken into account. The approach renders it possible to carry out numerical simulations of forced and parametric liquid sloshing in baffled tanks with baffles of different sizes and placed in different positions in the tank. This gives the possibility of governing the baffle radius and its position within the tank. It is very topical because practically the baffle effects can often be observed only after the baffle installation. The effects of parametric resonances are studied. The 
areas of unstable and stable liquid motions are obtained using the Ince-Strutt diagram. The proposed method makes it possible to determine the necessity of the baffle installation in tanks by using numerical simulation and thus to shorten the expensive field experiments. The proposed approach will be easily generalized to elastic tanks with elastic baffles. The geometry of tanks can also be changed, easily so the results will be obtained for conical, spherical and compound shells with and without baffles. It will allow giving recommendations about installation of protective elements (covers, partitions). As for the applicability limits of the proposed approach it should be noted that only the ideal incompressible liquid is under consideration, and the problems are formulated in linear statements.

\section{Acknowledgments}

The authors gratefully acknowledge our collaborator on the STCU Project \#6247, Professor Alex Cheng, University of Mississippi, USA for his constant support and interest in our research.

\section{Nomenclature}

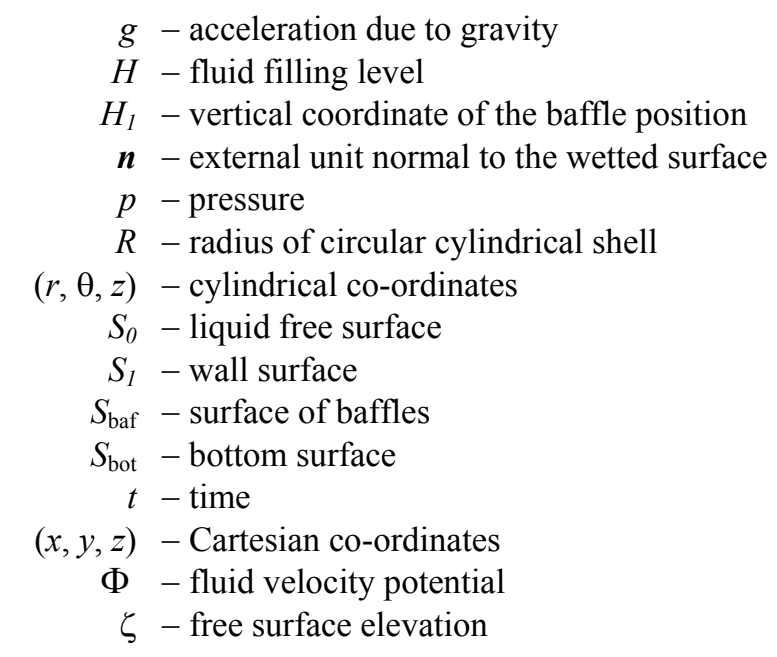

\section{References}

[1] Kumar R. (1971): Flexural vibrations of fluid-filled circular cylindrical shells.- Acoustica, vol.24, No.3, pp.137146.

[2] Biswal K.C., Bhattacharyya S.K. and Sinha P.K. (2004): Dynamic characteristics of liquid filled rectangular tank with baffles. - IE (I) Journal-CV, vol.84, pp.145-148.

[3] Abramson H.N. (2000): The dynamic behaviour of liquids in moving containers. - NASA SP- 106, Washington, D.C., 1966, updated by Dodge, F.T., Southwest Research Institute, pp.23-37.

[4] Ibrahim R.A. (2005): Liquid Sloshing Dynamics. Theory and Applications. - Cambridge University Press.

[5] Ru-De F. (1993): Finite element analysis of lateral sloshing response in axisymmetric tanks with triangular elements. - Computational Mechanics, vol.12, pp.51-58.

[6] Cho J.R., Lee H.W. and Ha S.Y. (2005): Finite element analysis of resonant sloshing response in 2-D baffled tank. - Journal of Sound and Vibration, vol.288, pp.829-845.

[7] Faltinsen O.M. and Timokha A.N. (2012): Analytically approximate natural sloshing modes for spherical tank shape. - J. Fluid Mech., vol.703, pp.391-401.

[8] Kim M.S. and Lee W.I. (2003): A new VOF-based numerical scheme for the simulation of fluid flow with free surface. Part I: New free surface-tracking algorithm and its verification. - International Journal for Numerical 
Methods in Fluids, vol.42, pp.765-790.

[9] Kim M.S., Park J.S. and Lee W.I. (2003): A new VOF-based numerical scheme for the simulation of fluid flow with free surface. Part II: application to the cavity filling and sloshing problems. - International Journal for Numerical Methods in Fluids, vol.42, pp.791-812.

[10] Strelnikova E., Yeseleva E., Gnitko V. and Naumenko V. (2010): Free and forced vibrations of the shells of revolution interacting with the liquid. - Proc. of XXXII Conference "Boundary elements and other mesh reduction methods", WITPress, Transaction on Modeling and Simulation, vol.50, pp.203-211.

[11] Gnitko V., Marchenko U., Naumenko V. and Strelnikova E. (2011): Forced vibrations of tanks partially filled with the liquid under seismic load. - Proc. of XXXIII Conference "Boundary elements and other mesh reduction methods" WITPress, Transaction on Modeling and Simulation, vol.52, pp.285-296,

[12] Choudhary N. and Bora S.N. (2017): Linear sloshing frequencies in the annular region of a circular cylindrical container in presence of a rigid baffle. - Sadhana-Academy Proceedings in Engineering Sciences, vol.42, No.5, pp.805-815.

[13] Gnitko V., Naumemko Y. and Strelnikova E. (2017): Low frequency sloshing analysis of cylindrical containers with flat and conical baffles.- International Journal of Applied Mechanics and Engineering, vol.22, No.4, pp.867-881.

[14] Wang J., Wang Ch. and Liu J. (2019): Sloshing reduction in a pitching circular cylindrical container by multiple rigid annular baffles. - Ocean Engineering, vol.171, No.1, pp.241-249.

[15] Miles J.W. (1958): Ring damping of free surface oscillations on a circular tank. - J. Appl. Mech., vol.25, No.2, pp.274-276.

[16] Gedikli A. and Erguven M.E. (2003): Evaluation of sloshing problem by variational boundary element method. Engineering Analysis with Boundary Elements, vol.27, pp.935-943.

[17] Brebbia C.A., Telles J.C.F. and Wrobel L.C. (1984): Boundary Element Techniques- Springer-Verlag: Berlin and New York.

[18] Gnitko V., Degtyarev K., Naumenko V. and Strelnikova E. (2018): Coupled BEM and FEM analysis of fluidstructure interaction in dual compartment tanks. - Int. Journal of Computational Methods and Experimental Measurements, vol.6, No.6, pp.976-988.

[19] Ravnik J., Strelnikova E., Gnitko V., Degtyarev K. and Ogorodnyk U. (2016): BEM and FEM analysis of fluidstructure interaction in a double tank.- Engineering Analysis with Boundary Elements, vol.67, pp.13-25.

[20] Wijngaarden Leen van. (2007): Prandtl-Batchelor flows revisited. - J. Fluid Dynamics Research, vol.39, pp.267-278.

[21] Gavrilyuk I., Lukovsky I., Trotsenko Yu. and Timokha A. (2006): Sloshing in a vertical circular cylindrical tank with an annular baffle. Part 1. Linear fundamental solutions. - Journal of Engineering Mathematics, vol.54, pp.71-88.

[22] Frenkel D. and Portugal R. (2001): Algebraic methods to compute Mathieu functions. - Journal of Physics A: Mathematical and General, vol.34, pp.3541-3551.

[23] Butikov E. (2018): Analytical expressions for stability regions in the Ince-Strutt diagram of Mathieu equation.American Journal of Physics, vol.86, pp.257-267.

Received: May 26, 2019

Revised: May 23, 2020 